\title{
Bridging the gap: interactive, case-based learning in radiology education
}

\author{
Mark D. Sugi ${ }^{1}$ [D $\cdot$ Tabassum A. Kennedy ${ }^{2} \cdot$ Vikas Shah $^{3} \cdot$ Michael P. Hartung $^{2}$
}

Received: 16 January 2021 / Revised: 6 May 2021 / Accepted: 22 May 2021 / Published online: 4 June 2021

(c) The Author(s), under exclusive licence to Springer Science+Business Media, LLC, part of Springer Nature 2021

\begin{abstract}
Traditional teaching methods in radiology education have not kept pace with advances in technology that foster successful transition into independent practice. This deficit has been exacerbated by the COVID-19 pandemic, as the need for social distancing and the introduction of hybrid staffing models have decreased the critical educational interactions at the reading room workstations between staff and trainees. By leveraging interactive, case-based learning, educators have the opportunity to bridge the substantial gap between basic pattern recognition and successfully making a diagnosis in independent practice. For the educator, this signals a shift away from perfect case selection and presenter authority, and toward the role of a guide facilitating an active case-based learning experience. This form of learning is best accompanied by guided interpretation and iterative feedback with the goal of developing similar levels of mastery and autonomy among graduating trainees. In this article, we present the tools and methods for incorporating interactive cases into existing and novel teaching materials to meet the unique challenges educators are facing today.
\end{abstract}

Keywords Interactive cases $\cdot$ DICOM $\cdot$ Abdominal radiology $\cdot$ Radiopaedia $\cdot$ Pacsbin

$\begin{array}{ll}\text { Abbreviations } \\ \text { DICOM } & \begin{array}{l}\text { Digital Imaging and Communications in } \\ \text { Medicine }\end{array} \\ \text { PACS } & \begin{array}{l}\text { Picture Archiving and Communication } \\ \text { System }\end{array} \\ \text { HIPAA } & \begin{array}{l}\text { Health Insurance Portability and Account- } \\ \text { ability Act }\end{array} \\ \text { COVID-19 } & \begin{array}{l}\text { Coronavirus disease } 2019\end{array}\end{array}$

\section{Introduction}

Traditional teaching methods in radiology education are largely based on techniques honed during the era of the viewbox with an emphasis on single-image pattern

Mark D. Sugi

mark.sugi@ucsf.edu

1 Department of Radiology \& Biomedical Imaging, University of California, San Francisco, 505 Parnassus Avenue, 3rd floor M391, Box 0628, San Francisco, CA 94143, USA

2 Department of Radiology, University of Wisconsin-Madison School of Medicine and Public Health, Madison, WI, USA

3 Department of Imaging, University Hospitals of Leicester NHS Trust, Leicester, UK recognition ("Aunt Minnie") and memorization of associated key facts. Despite many technological advancements allowing educators to more accurately simulate a diagnostic training experience in the lecture setting, the centerpiece of most didactic teaching remains a PowerPoint slideshow (Microsoft, Seattle, WA), which was released 34 years ago in 1987. While teaching radiographs fit well into this paradigm, this method fails to meet the unique educational challenges of effectively teaching the interpretation of cross-sectional imaging [1]. Specifically, the skills of organizing the study, sifting through hundreds or even thousands of images, comparing multiple anatomic structures, reviewing comparison studies, and finding key images that lead to a confident diagnosis cannot be adequately taught with traditional methods. These critical steps of solidifying lecture knowledge into practical application happen variably and independently without the supervision or expertise of the presenter to help guide and enrich the process.

Changes in staffing and education in response to the COVID-19 pandemic has further emphasized the need for educational innovation to bridge this gap. As the COVID19 pandemic began, strategies for social distancing among staff, trainees, and faculty necessitated moving towards hybrid or completely virtual learning and staffing environments [2]. This has resulted in a significant decline in the 
apprenticeship component of radiology education at the workstation, where faculty find new challenges in providing the experience of side-by-side staffouts and the irreplaceable value of observing the search pattern, organization, and thought process of the attending unfold while interpreting challenging cases. Social distancing has decreased the collective and spontaneous learning experience within a busy reading room that previously allowed all trainees the chance to benefit from the most educational cases of the day.

In this article, we describe methods to successfully transition the radiology educational model from the traditional emphasis on knowledge and pattern recognition to a modern paradigm emphasizing practical application and the skills needed for confident, independent practice.

\section{The value of interactive cases}

Interactive cases allow for the first true practical application of the material while still under the guidance of the instructor, essentially simulating the training environment of residency and fellowship while reinforcing core theoretical knowledge. In other words, the learner can be given the opportunity to make the diagnoses being taught by reviewing full cases before exiting the learning environment, rather than leaving it up to chance and potentially never truly reinforcing the concepts with real cases. Traditional curricula and methods aim to deliver breadth of knowledge; the model we describe adds the crucial dimensions of depth and practice.

In contrast, traditional lectures largely emphasize the expertise and skill of image selection of the presenter to convince the audience of the diagnosis being taught. Most practicing radiologists can recall a sense of uncertainty mixed with disillusionment when encountering less-convincing variations of the diseases they were taught so clearly during training. It is reasonable to suspect that many cases in journals and textbooks have been selected primarily based on how well they represent the disease process in a single image, rather than reflecting more common (but less pathognomonic) presentations, thus, overemphasizing the value of memorizing "Aunt Minnie" appearances for clinical practice.

Many educators recognize the gap that exists between lecture material and real-world clinical practice but may not be aware of the available tools or how to leverage them for a more immersive and interactive learning experience. This may be in part due to technological hurdles and the time investment associated with exporting, organizing, and presenting interactive cases rather than single images. There may also be pressure to "teach for the test" and follow the current format of the American Board of Radiology Core Exam. However, as these educational techniques gain traction, there may be increased interest in expanding scrollable and interactive cases in formal testing.

Fortunately, technological advances have lifted this restriction and allow educators to employ essentially any case of true pathology in the teaching setting irrespective of how well it displays in a few static images. In this learning environment, the risk of failure does not carry clinical implications and allows trainees to refine their mental representations of the disease process while further gaining knowledge [3]. Confounding factors that would normally be omitted from traditional lectures can be used to great effect, such as motion or artifactual degradation of image quality, and concurrent pathology that may tempt satisfaction of search. Interactive cases allow for the introduction of unexpected information into the learning process that commonly confounds clinical practice, encouraging learners to navigate cases of the same pathology from multiple perspectives. These methods promote the goals of mastery and autonomy, which together are essential for the transition to independent clinical practice [4].

In addition to complimenting traditional didactic teaching, focused pathology-based interactive case collections can enhance the training experience and provide remediation of common or critical misses. For example, if a resident is struggling with diagnosing closed-loop small bowel obstructions on call, they can be asked to review a collection of surgically proven cases to gain the necessary complex pattern recognition to confidently make the diagnosis that would be impossible to achieve by memorizing facts or annotated single images (Online Appendix 1). Additionally, learning curves can be applied to target the most efficient phases of learning during which the learner makes the most significant improvements in performance [5].

After overcoming some of the early technological learning curves, a whole new world of cases is opened up to the innovative educator; the reward for learning these technologies is lifting the heavy burden of "perfect" case selection from the educator's shoulders and shifting the focus from their expertise to facilitating an enriching, active learning experience for the participant as they learn firsthand from the cases. In the remaining sections, the authors will highlight the specific tools and techniques available to bring interactive cases to trainee education.

\section{Introduction to key tools and resources}

The development of electronic learning resources in radiology education is being driven by educators on a global scale [6] and has lowered the barriers to collaborating with our radiology colleagues in different parts of the world. Leveraging external resources on the web allows for broadening 
the scope of pathology outside a regional demographic and takes advantage of global, crowdsourced cases, including manifestations of rare diseases. It is a way to acknowledge, streamline, and organize the valuable contributions from passionate educators all over the world.

For the educator looking to build a personal DICOM teaching library or manipulate DICOM images for export to a variety of teaching platforms, the open-source, free image viewer Horos (Horos Project) for Mac OS is a powerful and essential tool (Online Appendix 2). Horos provides full PACS functionality, creation, and editing of new series and multiplanar reformats, and export options including JPEG, movie, and DICOM. A third-party plug-in enables automatic upload of cases from Horos into Radiopaedia (Online Appendix 3).

Creating an organized, robust personal teaching library using DICOM provides the greatest flexibility when considering novel teaching resources and removes a major stressor and time-consuming part of resource creation: finding the right cases. Busy clinical practices provide a wealth of educational cases that can be continually added to a growing teaching library, providing great flexibility for resource creation over time as the library grows. In the authors' experience, routine follow-up of complex or unknown cases often provides the most potent and insightful educational material and can often be efficiently accomplished using builtin features of the institutional PACS, for example, through follow-up alerts.
DICOM files can be exported in a HIPPA-compliant, anonymized fashion from their institutional PACS to their personal library for ready access for teaching opportunities. Some PACS-viewers allow users to direct export cases to an encrypted USB drive or network drive from their workstation, which can then be imported and organized in software like Horos. It is advisable to consult with Radiology Information Technology (IT) to determine the most efficient and institutionally compliant pipelines for exporting teaching cases. Potential hurdles can be overcome by emphasizing the value of their support and partnership in advancing new frontiers of radiology education.

Pacsbin (Pacsbin.com) is a web-based, fully featured DICOM-viewing platform that allows users to create a personal teaching library. It was developed by a radiologist and closely simulates a diagnostic PACS environment (Fig. 1, Online Appendix 4). The site performs automated anonymization at the time of the upload to ensure HIPAA compliance. Pacsbin has a highly intuitive user interface that functions well on laptops, tablets, and phones, usually requiring little explanation before users start reviewing cases. The mobile viewer maximizes screen real estate and provides a PACS-like viewing experience with window/level, zoom/ pan, and selection of different series, and works well to supplement a formal lecture as almost every attendee will have a smartphone available. Cases can be organized into lists and provided to trainees before or at the time of lecture in order to allow for personal interaction with the content being reviewed by the instructor. Links to these cases can

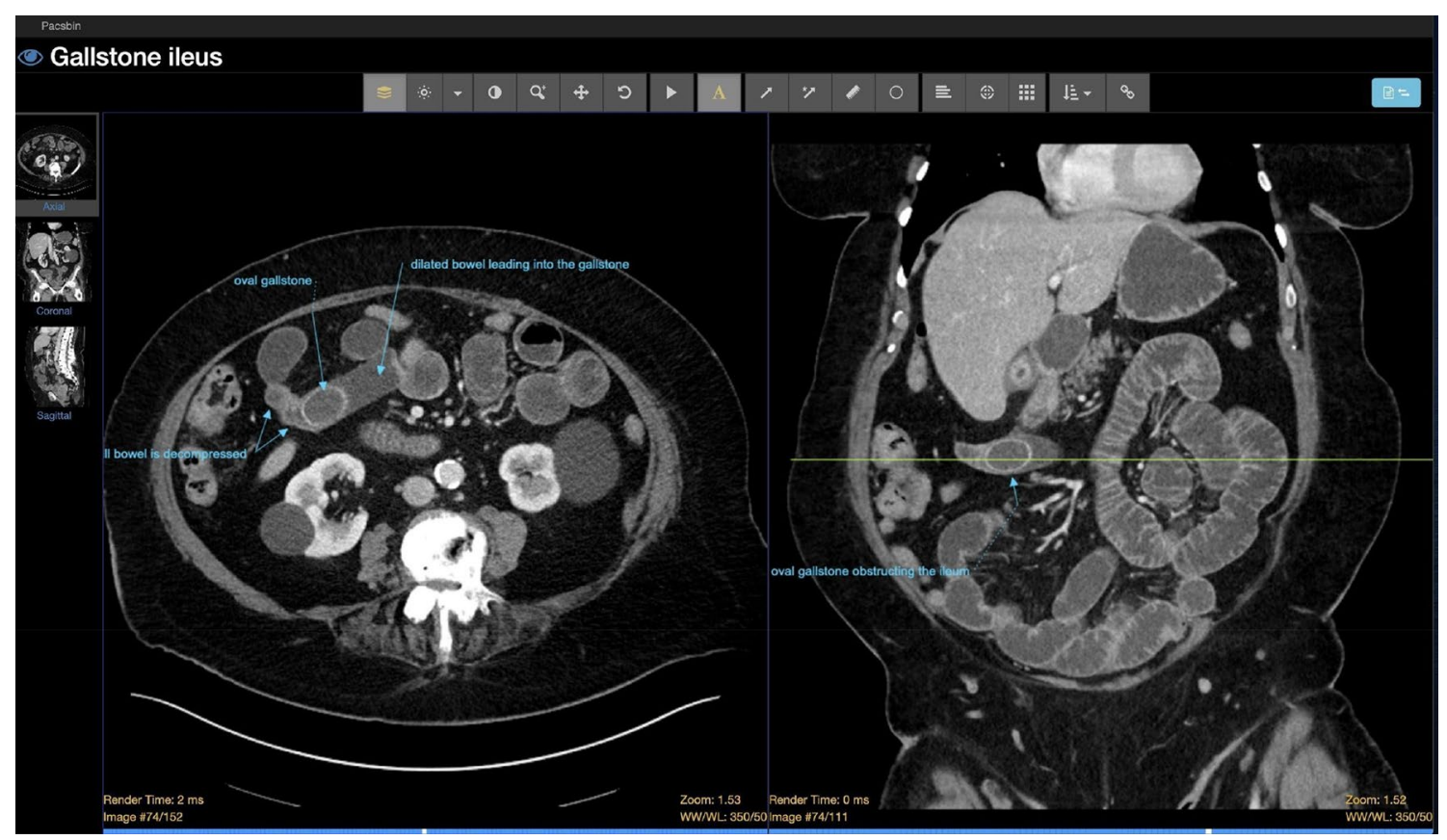

Fig. 1 Simulated PACS environment of Pacsbin (Pacsbin.com) allows for scrolling, windowing, and annotating multiplanar, anonymized interactive cases 
be readily shared with the audience using QR codes, which can be generated for free on the web and scanned using the phone's built-in camera app to rapidly open the case. Each account allows the first 20 cases for free, followed by small monthly fees for accounts with 500 or 2000 cases. There are also options for institutional licenses and integration of case upload/creation into the institutional PACS. ClariPACS (ClariPACS.com) has similar functionality but is less practical for the individual user due to substantially higher costs.

Radiopaedia (Radiopaedia.org) is a well-recognized free, wiki-based resource that is commonly referenced by trainees and practicing radiologists with about 41,000 cases and 14,800 articles. All contributions to the site are moderated by the editorial board to ensure accuracy, consistency, and relevance of content. The open-edit model encourages contributors from around the world to keep the resource up-todate, continue to add new cases, and write new articles. Individual users can contribute cases for the benefit of the public or keep them unlisted/private in a personal library. Currently supported formats include JPEG, PNG, and recently a beta option for DICOM with automatic anonymization. Personal and public cases can be organized into playlists that can be hidden or made available to the public, allowing for integration into lectures or for pre-classroom work assignments. A randomized, controlled evaluation of a medical student curriculum based on case playlists hosted on Radiopaedia demonstrated improved test performance among medical students when compared to traditional, lecture-based didactics [7].

Other web-based resources include the RSNA Medical Imaging Resource Center-Teaching File System (MIRCTFS), a web-based case database supported by user generated cases that can include static and scrollable images, and a more contemporary version under development, the RSNA Case Collection (cases.rsna.org). Additional educational websites emphasizing case-based review and interactive cases are provided in Online Appendix 5.

\section{Interactive cases for the presenter}

There are several ways to add interactive content to existing lecture materials that the presenter can review (Online Appendix 6). PowerPoint presentations can be supplemented with short video clips which can simulate a scrolling experience by dragging the progress bar. Many PACS allow for direct export of an image series to a movie file, which can be further edited with free tools such as ImageJ (NIH). Screen recording tools are widely available for free, including QuickTime Player on Mac and a built-in tool on Windows 10 , which can readily capture ultrasound cines or representative images of other cross-sectional studies. The principal limitations of video clips are file size and suboptimal scrolling capabilities.

Scrollable cases can also be shown in PowerPoint using the animate function but require the sequential images from the study to be dragged, dropped, and animated on the slide (Online Appendix 7). This creates an experience similar to a basic PACS-viewer and may be accompanied by an adjacent static image for reference on the same slide to guide the audience's attention toward the key findings. The limitations of this approach are that it is time consuming, results in large file sizes, and requires a PACS that facilitates export of JPEG images with the desired window/level setting or technical savvy with manipulating and exporting DICOM to JPEG with a program like Horos.

One of the benefits of Radiopaedia is that the entire case library can be leveraged as a resource to supplement traditional lectures using the Playlist function, allowing for a hybrid presentation of traditional slides with static images along with scrollable cases simulating a PACS experience (Fig. 2). The desired cases are added to a playlist, followed by the educational slides exported in JPEG format from a slideshow program such as PowerPoint or Keynote. The presentation can then be displayed directly from a web browser or downloaded for offline use as a perk for the highest level of supporter, which allows for faster loading times and does not require an internet connection (Online Appendix 8,9).

\section{Interactive cases for the audience}

There are several techniques for engaging the audience with interactive cases for the lecture setting. These can be considered before the lecture as "pre-work," during the lecture under the guidance of the instructor, or available as supplemental materials at the conclusion of the lecture for solidifying concepts. The cases can be prepared and organized in a Radiopaedia playlist, Pacsbin collection, custom webpage, or another format. Learners can access the cases via weblink shared with email or on an introductory slide, a public website, or QR code (Fig. 3). QR codes are particularly effective for sharing cases "on the fly," as they can be quickly scanned with a phone's built-in camera app which opens a weblink to the case and allows for review on a personal device.

An example of pre-work is sending 3-5 representative cases of liver pathology via email that will be discussed in greater detail during the formal lecture the following day. The presenter may consider an accompanying quiz to gage the audience's knowledge level and appropriately pace and focus the lecture. During the lecture, the learner can be given the option to review cases along with the presenter by linking cases to QR code or weblink (Online Appendix 10). For early learners such as medical students 
Fig. 2 Radiopaedia full-screen mode viewing platform, which can be integrated into lectures between slides to allow for guiding learners through realtime interpretation of an actual case. Case details can be hidden from the learner by selecting the "hidden diagnosis" link

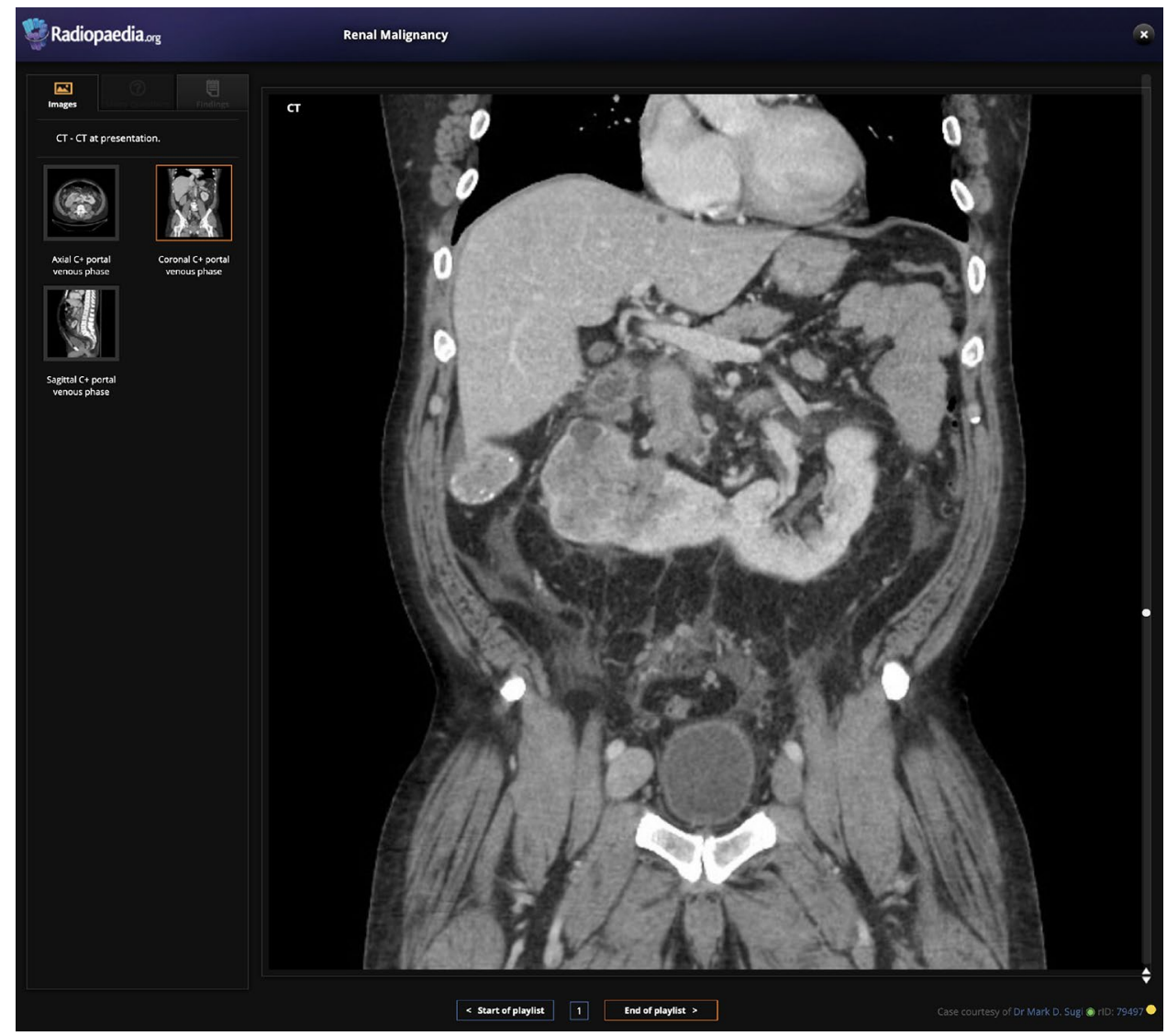

or non-radiologists, this method is particularly engaging and effective and can be the basis of entire didactic sessions for teaching the basics of using a PACS viewer, identifying basic anatomy and search patterns, and learning the features of common pathology. Indeed, a medical student summarized the strengths well when providing feedback for an abdominal CT conference using these methods: "Having the chance to read scans ourselves under supervision was awesome. I feel a lot more comfortable looking at abdominal CT than I did before this session." (Online Appendix 11).

For trainees and practicing radiologists, cases can be linked out with QR codes and reviewed along with the presenter, or given 1-2 min for personal review before group discussion. This format can also foster collaboration and discussion with colleagues, possibly dividing the group into teams to build camaraderie and stimulate a positive sense of competition. Different variations can be tested and introduced into the traditional lecture setting to increase engagement and challenge learners of all levels, as properly selected cases will have valuable teaching points for all levels of trainees as they scroll through the material and interact at their training level. For example, a medical student might focus on learning the basic anatomy, while a senior resident focuses on refining their differential diagnosis.
At the conclusion of the lecture, the cases and supplemental material can be made available for the learner via weblink displayed on a summary slide, QR code, or hosted on a custom webpage. This will allow them to revisit the material and take additional time as needed to solidify key teaching concepts, rather than facing uncertainty at the next chance encounter of the pathology when they are on their own.

Engagement and assessment of understanding during the presentation may be evaluated in a number of ways. The simplest means of observing body language have been made difficult with the pivot to online education accelerated by the COVID-19 pandemic. Most web-based meeting platforms have a chat box which can be used to encourage discussion. Integrating audience response software such as polling may also be considered. A plethora of such platforms are available; the authors have the most experience with Poll Everywhere (polleverywhere.com) which allows question types such as multiple choice, open text which can be displayed as word clouds, or selecting regions on images, similar to that used in Diagnosis Live by RSNA. Many polling platforms offer plug-ins to allow the polls to be seamlessly integrated into PowerPoint or Keynote presentations. 
Fig. 3 Use of a QR code in a lecture slide to provider learners with a quick reference to interactive cases that can be accessed via laptop, tablet, or phone during the lecture. A larger version of the $\mathrm{QR}$ code is included, and readers are encouraged to test scanning it with their phone's camera app to open the case

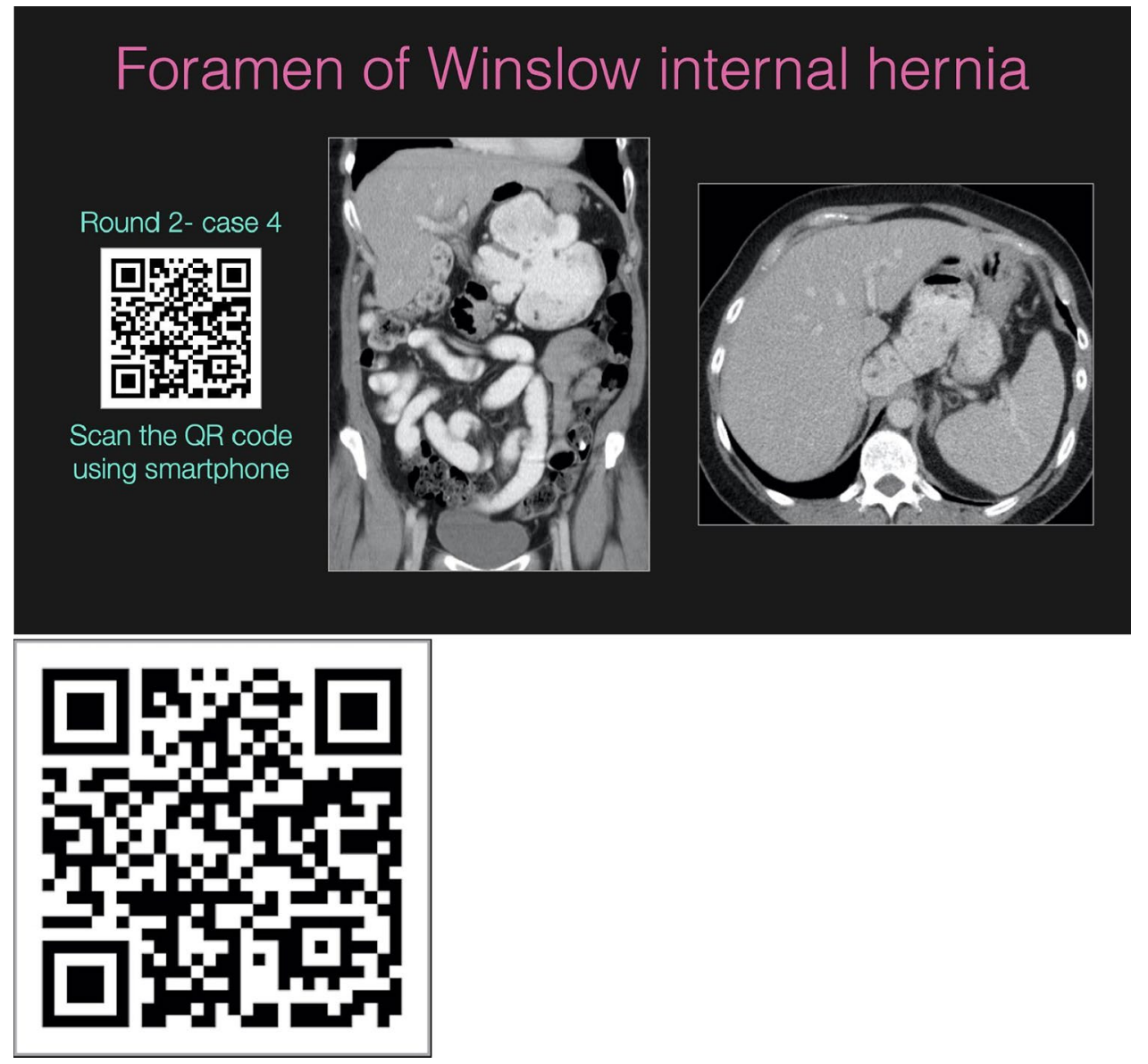

\section{Conclusions}

Traditional radiology teaching methods have not kept pace with advancements in technology and leave a substantial gap between knowledge acquisition and study interpretation. Interactive, case-based learning provides an opportunity to bridge this gap and prepare trainees for real-world clinical practice. A multitude of tools is available to enhance and augment the didactic teaching provided by the tutor with full imaging studies in simulated PACS environments, adding depth of hands-on experience to breadth of knowledge.

Supplementary Information The online version contains supplementary material available at https://doi.org/10.1007/s00261-021-03147-z.

Authors contribution Conceptualization: MDS, MPH. Writing-original draft: VS, MPH. Data curation, writing — reviewing and editing: All authors. Supervision: MPH.

\section{Funding None.}

\section{Declarations}

Conflict of interest Hartung-Editor, Radiopaedia. Shah-Senior Editor, Radiopaedia.

\section{References}

1. Felson B, Jacobson G, Pendergrass EP, Bristol LE, Linton OW, Harrington RW. Viewbox seminar: a new method for teaching roentgenology. Radiology 1975;116:75-8.

2. Mossa-Basha M, Meltzer CC, Kim DC, Tuite MJ, Kolli KP, Tan BS. Radiology Department Preparedness for COVID19: Radiology Scientific Expert Review Panel. Radiology 2020;296:E106-12.

3. Ericsson KA. Acquisition and Maintenance of Medical Expertise. Acad Med 2015;90:1471-86.

4. Petersen RY. Taking Mastery Learning One Step Further. Acad Med 2016;91:751.

5. Pusic M, Pecaric M, Boutis K. How Much Practice Is Enough? Using Learning Curves to Assess the Deliberate Practice of Radiograph Interpretation. Acad Med 2011;86:731-6.

6. Oleaga L, Dewey M, Iezzi R, Kainberger F, Nyhsen CM, Catalano C, et al. ESR statement on new approaches to undergraduate teaching in Radiology. Insights Imaging 2019;10.

7. El-Ali A, Kamal F, Cabral CL, Squires JH. Comparison of Traditional and Web-Based Medical Student Teaching by Radiology Residents. J Am Coll Radiol 2019;16:492-5.

Publisher's Note Springer Nature remains neutral with regard to jurisdictional claims in published maps and institutional affiliations. 